\title{
Bruchstück eines altfranzösischen Mystère.
}

Von dem Direktor des Staatsarchivs zu Münster, meinem verehrten Kollegen Herrn Professor Dr. Philippi, wurden mir vor kurzem sechs lose Blätter freundlichst zur Einsicht überlassen, die er in der Bibliothek des dem Fürsten Salm-Salm gehörenden Schlosses Anholt gefunden hatte. Sie sind ein Bruchstück eines etwa zu Ende des fünfzehnten Jahrhunderts auf Papier niedergeschriebenen altfranzösischen Textes und zwar eines Mystère in Versen, das den Märtyrertod der Apostel Petrus und Paulus behandelt. Auf Grund einer genauern Betrachtung dieser sechs Blätter darf ferner angenommen werden, dafs sie zu einem Texte gehört haben, der zu einem nicht geringen Teile auf eine gemeinsame Vorlage mit dem Stücke zurückgeht, das bei Jubinal, Mystères inédits du XVe siècle (1837) I, 6r ff. nach der Handschrift der Bibliothek Sainte-Geneviève zu Paris abgedruckt und von Petit de Julleville, Les Nystères (1880) II, 546 näher besprochen ist.

Nachdem ich dies festgestellt hatte, gelang es mir ohne grofse Mühe Ordnung in die Reihenfolge der Blätter zu bringen. Es ergab sich, dafs wir es mit zwölf schmalen einspaltig beschriebenen Seiten zu thun haben, von denen jedoch die sechs letzten zum Teil arg verstümmelt sind.

Die Uebereinstimmung mit Jubinal reicht zunächst aber auch nicht weiter als etwa bis zur Mitte der fünften Seite; erst auf der zehnten Seite findet sich wieder eine Stelle, die bei Jubinal etwas Entsprechendes hat. Wie es sich mit dem übrigen Teile des Bruchstücks verhält vermag ich nicht $z u$ sagen. In seiner Besprechung der Actes des Apôtres par Arnoul et Simon Greban führt Petit de Julleville (a. a. O. 46I) drei Ausgaben dieses Dramas an. Die an erster Stelle von ihm genannte Ausgabe aus dem Jahre 1538 habe ich einsehen können: die Göttinger Bibliothek besitzt ein Exemplar derselben, das ich mir nach Münster kommen liefs. Leider überzeugte ich mich bald, dafs dieses Mystère nichts dem Anholter Bruchstück Entsprechendes darbietet. Ob dies vielleicht bei dem andern Mystère de Saint-Pierre et Saint-Paul, das Petit de Julleville a. a. O. 548 behandelt, der Fall ist, mufs ich dahingestellt sein lassen, da mir die Ausgabe desselben nicht zugänglich war. - 
Dic Schrift des Bruchstücks ist vielfach an und für sich so undeutlich oder doch so verwischt, dafs eine Entzifferung des Textes nicht immer möglich ist. Nicht selten hat sich auch von einer Zeile nur ein einziges Wort, mitunter nicht einmal ein Buchstabe erhalten. Andrerseits konnte ich jedoch an mehreren Stellen, die dem Verständnis zuerst ganz besondre Schwierigkeiten boten, eine befriedigende Lesart dadurch erzielen, dafs Herr Archivdirektor Philippi mir seine thätige Mitwirkung zu teil werden liefs, wofür ihm hier der herzlichste Dank abgestattet sei.

Ich hatte die Arbeit bereits abgeschlossen, als ich durch die kurze Notiz Gröbers in der Zeitschrift für romanische Philologie XXV. Band, 1. Heft S. 128 auf die dankenswerte Dissertation von Julius Poewe (Halle I 900$)^{1}$ aufmerksam wurde. Sie hat mir für einige Stellen gute Dienste geleistet.

Das Mystère bei Jubinal beginnt mit einem Gespräche zwischen Petrus und römischen Bürgern. Der Apostel sucht diese für das Christentum zu gewinnen. Gegen ihn tritt auch Clemens auf, der sich später bekehrte und Papst wurde. In der Rede des Letztern haben wir den Anfang des Anholter Bruchstücks vor uns; die Uebereinstimmung mit dem Text bei Jubinal ist oft wörtlich.

Jubinal I, $63-68$. (A).

Anholter Bruchstück. (B).

S. Clement, escolier.

Or est tout nient, car s'il avoit

Celle puissance qu'avez dite,

Il eust este de la mort quite;

Car par quelle voye mourroit

5 Qui Dieu seroit, qui tout pourroit?

Voir s'il mourut et trespassa,

La mort sa deite quassa

Et son povoir ly fu tollu.

\section{S. Pierre.}

Mon bel amy, s'il eust voullu,

Io Bien se feust garde de mourir,

Mais a nostre mort secourir

Nostre sire usa par sagesce

$D \in$ merveill(i)euse soutillesce

Contre la cruele malice

15 De l'anemy plain d'injustice;

Car il voult homme devenir

Et nos miseres soustenir,

Que de bon ... il nous amoyt (f. Ir)

Et pour nostre amour trespassa;

La deite sa mort cass[a]

Et son povoir luy fut tolli.

Petrus.

Mon bel amy, s'il l'cust voulu,

Bien se feust garde de morir;

Mais pour nostre mort secourir

Usa par sa tresgrant largesse

De merveilleuse subtilesse

Contre la cruelle malice

De l'ennemy tout plain de vice; 15

Car il voult homme devenir

Et noz miseres soustenir

I s'il avoit nämlich Christus.

1 Sprache und Verskunst der Mystères inédits du XVe siècle (abgedruckt von A. Jubinal, Paris 1837). 
A celle fin que l'anemis,

Qui homme avoit souz le pie mis,

Sy fust par homme sourmonte

Et sa mauvestie par bonte,

5 Et mort par mort a mort livree;

Et se l'ame fu[ $t]$ dessevree

Du corps selonc l'umanite,

Nient mains avoit la deite;

Tout povoit immutablement

10 Qui les rassembla dignement

Et resuscita home et Dieux,

Puis monta puissaument au[ $\mathrm{x}]$ cieux.

$\mathrm{La}$ en ame et en corps yront

Ceulz qui de cuer le serviront

I5 En joye, en doulceur, en seurte,

En pardurable beneurte;

Mais ceulz qui en $[$ [u]y ne croiront

Ou qui son vouloir ne feront

Yront en tourment pardurable.

Symon l'enchanteur.

20 Seigneurs rommains, c'est bourde et fable

Quant que ce vilain va disant.

Croirez vous qu'un povre paisant

Qui fut pendu puisse estre Dieux?

En vous devroit crever les yeux.

25 Moy, moy par qui honneur avez,

Qui fais vertus, vous le savez,

Devez aourer et m'obeir.

\section{S. Pierre.}

Symon, mais on te doit heir

Qui fais injure et vilenie

30 A celuy qui te donna vie;

Mais tu es tout plain de pechie, Sy n'ist de toy fors mauvestie:

Tes diz sont envenimemens,

Tes fais ne sont que enchantemens,

$35 \mathrm{Ta}$ vie actrait la maise mort.

6 Die Hs. hat fut nach Poewe 74. 12 l. puissanment. 22 s. Anm. 23 Diex. 24 yeulz. 27 zu lang; B scheint hier das Richtige zu bieten, wenn nicht aourer als zweisilbig zu fassen ist (Poewe 46). 34 l. qu'en. chantemens.
Contre ces malves ennemis

Qui avoi(s)t home mort porquis

Si fut par home surmonte

De sa mauvaistie par bonte,

Car sa char fut a mort livree

Et l'ame si fut separee

Du corps selon l'umanite,

Et neantmoins la deite

Qui tout peut trespuissantment

Et les ressembla dignement

Et ressucita homme et Dieulx,

Puis monta puissamment es ci[eu]lx.

En corps, en ame cilx yront

Qui de bon cuer le serviront

En joie et en seurete

Mais celx qui en luy ne croiront

Ou qui son vouloir ne feront

Yront en torment pardurable.

Symon Magus loquitur.

Seigneurs romains, ce n'est que fable 20

Se que ce villain va disant.

Et pencez vous que ung tel paisant

Qui fut pendu puisse estre Dieulx?

On vous devroit crever les yeulx.

Moy, moy qui pour honeur aves 25

Qui fais vertu, vous le saves,

Deves aymer et obair.

\section{Petrus.}

Symon, maiz on te doit hair

Qui dis injure et villanie

A celuy qui te donna vie;

Maiz tu es tout plain de pech[i]e,

Se n'est que de ta mauvestie.

Tes diz sont tous envellimeurs

Et tes faiz sy sont enchanteurs,

$\mathrm{Ta}$ vie atrait mauvaise mort.

2-6 sind verderbt, während der Text in A verständlich ist. 9 ist um um eine Silbe zu kurz; vielleicht $Q u z$ tout peut out trespuissantment. 13 yront wird kaum aus $y$ vont entstellt sein, vielmehr A das Richtige bieten. In der Ueberschrift hat die Hs. maius. 22 1. qu'ung. 25 qui pour wohl Schreibfehler für.par qui. 32 Es könnte auf Grund von $A$ gelesen werden $S i$ n'ist que de ta mauvestie; aber A hat Besseres. 
Pausa. Les senateurs de Rome mainent Pierre et Paul et Symon Magus a ung home mort pour le faire ressuciter et quant il luy

\section{Marcel.}

Seigneur, vecy .I. homme mort.

Resuscites le sy verrons

Lequel dit voir sy croirons

Que celuy soit Diex en verite

5 Par qui sera resuscite;

Se non tous .II. le conparrez.

$$
\text { Symon. }
$$

Tout en l'eure vif le verrez.

Lors die aucune rien au mort en l'oreille et le mort remue la teste sans soy bougier.

Le second bourgoys.

Esgar[de], il remue la teste:

Pierres, vous mour $[r]$ ez comme beste;

Io Nous vouliez vous decevoir?

S. Pierre.

Attendez, vous sarez de voir

Se le mort ara mort ou vie.

$$
\text { Symon. }
$$

Sanglant vilain, fol, plain d'envie, Ne l'as tu pas veu remuer?

$$
\text { S. Pierre. }
$$

I5 Il ne fault plus contrarguer:

s'il vit, boive et menjusse et voise.

\section{Symon.}

Pierres, tu quiers tousjours la noyse;

Tu t'en pourras bien repentir:

Chascun puet et veoir et sentir 2o Que homme mort ne se puet bougier.

3 zu kurz. Poewe (60) möchte le vor voir einfügen, aber es wird zu lesen sein sy le croirons. Vgl. B. $4 \mathrm{zu}$ lang; vielleicht ist verte zu lesen; vgl. Poewe 56. In der Bühnenanweisung stehen in der Hs. (Poewe 74) hinter bougier noch die Worte die ly qu'il remue la teste sans soy bougier. 20 l. Qu'homme.
Pausa. fait ung tour d'ung baston autour Une du mort en disant: grisogoulus figure. guligoleste.

Regarde(s), il remue la teste:

Pierre, vous mourres come beste;

Nous cuides vous tous decepvoir? Io

\section{Petrus.}

Atendes, vous saures de voir,

Ce le mort ara mort ou vie.

Simon Magus.

Truant villain, tout plain d'envie,

Ne l'as tu pas veu remuer?

Petrus.

Il n'y fault plus contralier:

Cil ne vit et mengue et boive.

Simon Magus.

Pierre, tu n'as toujours que ....

Tu t'en pourras bien repentir:

Chacun peu(s)t bien voir et sentir

Que homme ne ce peult remuer 20

In der Bühnenanweisung wird luy in $y \mathrm{zu}$ bessern sein. 31 . si le croirons. In der Bühnenanweisung scheint ein Verseben des Schreibers vorzuliegen, denn die Worte et fait ung tour u. s. w. beziehen sich doch wohl auf Simon Magus. 16 ist verderbt. 17 Hs. touriours. Diese drollige Schreibung kommt in dem Texte noch mehrere Male vor. Die letzten Buchstaben der Zeile sind nicht zu entziffern. 20 ist verderbt, wie schon der Reim zeigt. 
S'il ne puet boire ne mengier,

Puis qu'il se muet, qu'en ay je a faire?

S. Pierre au[x] bourgoys.

Seigneurs, faictes lay en sus traire;

Sy verra on s'il yra point.

$$
\text { S. Clement. }
$$

5 Or vient bien cet debat a point.

Maistre Symon, traiez (vous) arriere!

Nous concluons par tel maniere

Que s'il ne va, Pierres, sachiez,

Il convient qu'aler le faciez

Io $\mathrm{Ou}$ autrement vous $\mathrm{y}$ mour $[\mathrm{r}] \mathrm{ez}$.

$$
\text { S. Pierre. }
$$

Et se aler puet que me donrez?

Vous ne me prometes que paine.

$$
\text { S. Clement. }
$$

Vostre creance toute plaine

Tout pleinement, sire, croirons,

15 Et maistre Symon punirons

Ainssy qu'il veult qu'en vous punisse.

$$
\text { S. Pierre. }
$$

Le mort boug[e] il janbe ne cuisse?

Regardez comment il se porte.

Marcel, en le tastant.

Par Mahommet, sa teste est morte; 20 Il n'ot, il re muet n'il ne rit.

\section{S. Pierre.}

Or pert (il) bien que malvez esp(e)rit Vous a fait une illusion.

Ainssy meine a confusion,

Ainssy detient, ainssy enlace

25 Ceulz a qui Dieu soutrait sa grace

Par leur pechie et desmerites.

Le second bourgoys.

S'il est ainssy comme vous dites

Faictes tost revivre ce mort.

Saint Pierre, a genous.

Doulz Dieu, qui de l'amere mort D'enfer gestates homme et fame, 30 Vueilliez remettre en ce corps l'ame.

$$
\text { u. s. w. }
$$

3 wird luy statt lay zu lesen sein; vgl. 6.6 vous kann entbehrt werden; vgl. Poewe 65. I I 1. s'aler. 20 muit. $21 \mathrm{Vgl}$. Poewe 65 und 57. An der letztern Stelle wird gezeigt, dafs esperit bald drei-, bald zweisilbig steht.
Ne ne puet boire ne mengier;

Puis qu'il remue qu'en ai ge a faire?

Petrus.

Seigneurs, faictes le sus piez traire

Sy verra l'en s'yl ira point.

Clemens.

Or vient ceste raison a point.

Maistre Symon, alles arriere!

Nous concluons par tel(le) maniere

Que se ne va, Pierres, saich[i]es,

Il ... qu'aller le facies

Ou autrement vous y mourr[es]. 10

$$
\text { Petrus. }
$$

Et ce aller peult que me dorres?

Vous ne me promectes que paine.

Clemens.

Vostre creance toute plaine

Tout plainement, sire, croirons,

Et maistre Simon pugnirons

Ainxi qu'il veult qu'on vous pugnice.

\section{Petrus.}

Le mort ne bouge bras ne cuisce;

Regardes coment il se po(u)rte.

\section{Marcellinus.}

Par Mahomet, sa teste est morte;

Il n'oyt, il ne meult, il ne rit.

Petrus.

Il pert bien que maulves esp(e)rit

Vous a fait une illusion;

Ainxi maine a confusion,

Ainssi dessoit, ainssi enlace

Celx a qui Dieu surtrait sa grace 25

Par leur faiz et vices mauldites.

Secundus burgenssis.

S'il est tout ainssi que vous dictes,

Faictes tost revivre se mort.

Saint Pierre s'en va a coste et se met a genoulx et fait sa priere

a Dieu et dit Petrus:

Doulx Jhesucrist qui de l'amere mort

...$\cdot$.,$\cdot$.

30

2 scheint remue aus se muet entstellt zu sein. 4 sy lira. I I l. c'aller. 24 enlaxe. 29 Thesucrist ist wohl Schreibfebler für Dieu, wie $\mathbf{A}$ bietet. 
Das von Gott erflehte Wunder gelingt: der Tote erwacht, worauf die Bürger an den Zauberer Simon Hand anlegen wollen, aber auf die Fürsprache des Apostels hin davon ablassen. Simon beschwört nun seinerseits die Teufel, ihm Kraft zu verleihen frei durch die Luft zu fliegen. Als dies geschieht, wissen die Bürger sich vor Erstaunen nicht zu lassen, Petrus aber fleht zu Christus dem Treiben der bösen Geister Einhalt zu thun. Sein Gebet wird erhört, der Zauberer fällt zu Boden. Mit dessen Klage über sein schweres Mifsgeschick und seine Ohnmacht dem Christengott gegenüber beginnt die Uebereinstimmung beider Texte aufs neue.

\section{Jubinal I, $71-72$.}

$$
\text { Symon. }
$$

Ahay Jhesucrist! trop es fort;

Contre toy ne vault nul effort.

Tu m'as trop lourdement coyssy:

Je suis tout ronps et tout froyssy.

$5 \mathrm{Je}$ ne puis aler ne [courir],

De male mort me fault mourir,

Ou feu d'enfer m'en fault aler.

Cy face le mort.

Le premier diable.

Ha, ba! Symon, or du baler!

Maistre Symon, sire Symon,

10 Vostre corps qui est de limon

Vouloit voler lassus au ciel!

Il desplaisoit a dan Michiel

Sy estes trebuchie a honte;

Car bas doit cheoir qui trop hault monte.

I5 Ou puis d'enfer vous porteron.

$$
\text { Le second. }
$$

Ta, ta, Symon, l'amy Neron!

Ton orgueil, ton enchanterie,

Ta mauvestie, ta simonie

Te seront bien tost chier vendus! 20 Passe! tu es nostre rendus.

Cy l'emportent hors du champ en uslant.

4 ronps muls fehlerhaft sein; vielleicht ist dafür roupt $z \mathrm{u}$ lesen oder unter Tilgung des zweiten tout die Form ronpu einzusetzen. 5 courir fehlt in der Hs. (Poewe 74). I4 cheoir ist einsilbig zu lesen; vgl. Poewe 43.

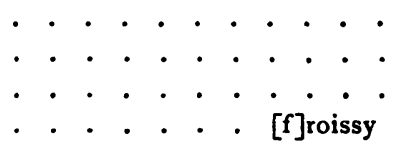

Je ne puis aller ne venir (f. $2^{r}$ ) 5 De malle mort me fault mourir, Ou puis d'enfer me fault aller.

\section{Satan.}

Ha, ha, Simon, or du voller!

Maistre Simon, sire Simon,

Vostre corps qui est de limon

Vouloit voller lassus au $c[i]$ el!

Il desplaisoit a saint $\mathrm{Mich}[\mathrm{i}] \mathrm{el}$

Si estes trabuche a honte;

Car a bas chiet qui trop hault monte.

Ou feu d'enfer vous trametron. Burgebus.

Or sa, Simon, l'amy Noiron!

Ton orgueil, ton enchanterie

Ta mauvaistie, ta simonie

Te seront chierement vendus!

Passe! tu es nostre rendus.

Die folgenden Verse werden vom Anholter Bruchstück allein geboten; im Text bei Jubinal findet sich nichts Entsprechendes. 
Leviatem.

Vous boullires tout au dessus

De nostre maistre Lucifer

Dedens la chaudiere d'enfer;

La aures vous de grans assaulx

5 De colevres et de crapaulx

Et serpens qui vous guerreiront

Et les menbres vous aracheront;

La souffrires paine et torment.

Astarot.

Tormente seres aultrement,

Io Foi que je doy a Lucifer:

Je vous mectray . . chaudement

Au plus parfont du puis d'enfer

- En poix en plom huille bollie;

Je vous boulleray sans cesser,

15 Toujours vous tiendray compaignie

Si n'aurez besoing de chanter.

Les diables portent Simon Magus en enfer,

criant et breant, et Lucifer dit:

Or sa! vous soies bien venus!

Qu'il me soit despoulle tout nus

Et le me boutes chaudement

20 En plom et en huille boullant;

Gardes que le tormenties bien,

Car onc en sa vie ne fit bien.

Les diables dient tous ensemble:

Lucifer, ne vous soucies [rien],

Nous le gouverneron tresbien.

Jubinal I, 72-74.

S. Clement.

25 Chier sires, or veons nous bien Que nostre loy sy ne vault rien. Sy la voulons du tout lessier.

S. Pierre.

Il vous fault doncques baptisier.

S. Clement.

Et que vault tel baptisement?

S. Pierre.

30 Beau frere, par l'arousement

Qu'en fait d'yaue par dehors,
Pausa. Clemens.

Cher sire, or voions nous bien

Que nostre loy si ne vault rien.

Si la voulons du tout laisser.

Petrus.

Il vous fault doncques baptizer.

Clemens.

Et que vault ci baptisement?

\section{Petrus.}

Beau frere, par l'arousement

$7 \mathrm{zu}$ lang; vielleicht ist vous $\mathrm{zu}$ tilgen oder trencheront statt aracheront zu lesen. II Nach mectray scheint bien zu stehen, ist aber nicht genau mehr zu erkennen. 13 Vielleicht: En poix, plom et huille bollie. 15 touriours. $22 \mathrm{zu}$ lang; l. Qu'onc.

3I wird yaue schwerlich, wie Poewe (50) meint, als dreisilbig ge31 s. Anm. 
En la getant desus le corps,

De tout pechie, soit veniel,

Ou mortel ou originel,

Dieu par dedens l'ame netoye.

5 Et grace ly donne et octroie,

En tant que se l'omme mouroit

En tel estat s'ame en iroit

Sans paine et sans faire sejour,

Plus clere et plus belle que jour

Io En la joye de Paradis.

S. Clement et les autres.

$\mathrm{Ne}$ soyez, sire, plus tardis!

En Dieu croions, baptisiez nous!

S. Pierre.

Or alez trestous a genous!

Cy voisent a genous.

Lors les baptise en disant:

En la fourme de sainte Eglise,

15 Mes bons amis, je vous baptise,

In nomine Patris et Filii et

Spiritus sancti. Amen.

S. Pierre a S. Clement:

Clement, nostre chier filz en Dieu,

Vous tendrez apres moy mon lieu;

Des maintenant vous y ordene,

Et pour Dieu, chier filz, metez

paine

20 De faire a Dieu plaisant servise.

Preschiez la loy de sainte Eglise!

Les non croians convertissiez

Et les non sages enseigniez,

Aux saintes gens honneur portez

25 Et les imparfais supportez;

Soiez de tout bien examplaire!

\section{S. Clement.}

Saint Pere, je suis prest de faire

La Dieu volente et la vostre.

$\mathrm{S}$. Pierre, en ly metant la main sus la teste:

Et du povoir Dieu et du nostre 30 Vous donnons papal dignite

Et nostre plaine auctorite.

sein entweder Que l'en fait d'yaue par dehors oder Qu'en fait de l'yaue par dehors.
En la gectant dessus le corps, $\mathrm{Ne}$ (s)toie tout peche mortel,

Soit grant, petit ou veniel.

$\begin{array}{llllllllll}\cdot & \cdot & \cdot & \cdot & \cdot & \cdot & \cdot & \cdot & \cdot & \cdot \\ & \cdot & \cdot & \cdot & \cdot & \cdot & \cdot & \cdot & \cdot & 5\end{array}$

En . . . . . . . . . (v)

En cel estat s'ame . . . .

Sans paine, sans fere sejour,

Et sans souffrir jamais dollour

Ira tout droit en Paradis.

Clemens.

Sire, n'en soies plus tardis!

En Dieu croiron, baptises nous!

Saint Pierre prent de l'eaue et dit:

Or alles trestous a genoulx!

En la forme de saint[e] Eglise,

Mes chers amis, je vous baptise 15

Ou nom du pere et du filx

Et aussy du saint esperit.

Clemens, nostre chier filz en Dieu, Vous tendrez apres moy mon lieu;

Des maintenant vous $y$ ordene, 20

Et pour ce, chier filz, mectes paine

De fere a Dieu plaisant servise.

Prechiez la foi de sainte Eglise!

Les non(s) creans convertisses

Et non(s) sa[i]ves vous enseignes, 25

Et aux sainctes honneur portes;

Les inpaciens $s u(r)$ pourtes

Et faictes tous bon exemplaire.

Clemens.

Saint Peres, je suis prest de faire

$\mathrm{La}$ voulente Dieu et la vostre. $\quad 30$

Saint Pierre luy baille les vestemens de sainte Eglise et le reve(i)st come pape et luy dit:

Du povoir de Dieu et du [nostre]

Vous donnons papal dignite

De nostre plaine auctorite,

7 En ce lestat. 9 dolleur. $\quad 16$ und 17 scheinen verderbt $z u$ sein, da das $e$ von pere im Hiatus steht und der Reim ungenau ist. 19 teandres. 20 ordonne. 
Le Saint Esp(e)rit sy vous parface Et le saint esprit vous parface

En tout bien et en toute grace. In nomine Patris et Filii et Spiritus sancti. Amen.

Lors se sieent a terre $\mathrm{S}$. Pierre et S. Pol. Titus et Lucas et S. Clement et les bourgois voisent en leur logeis.

En tout bien et vous doint sa grace.

In nomine Patris et Filii et Spiritus sancti. Amen.

Clemens.

Saint Pere, je vous re[n] grace

De l'onneur qu'il vous plaist me faire.

$$
\text { Petrus. }
$$

Entendes toujours a bien faire

Et a servir nostre seigneur.

Pausa.

3 zu kurz; vielleicht je vous ren

ci oder bien grace. 5 touriours.

Im Text bei Jubinal tritt nun Nero auf und bespricht sich mit seinen Ratgebern über die gegen die Apostel und deren Anhänger zu ergreifenden Mafsregeln. Einige von den letztern suchen die Apostel zu bewegen Rom zu verlassen und so den fernern Nachstellungen Neros zu entgehen. Den vereinten Bitten des Titus, Lucas und Clemens giebt Petrus endlich nach. - Im Anholter Bruchstück dagegen schliefsen sich an das Vorhergehende folgende Verse an:

\section{Marcellinus.}

Mallaquin, il te fault aller

Devers l'emperiere de Romme

Pour luy deviser et monstrer

Le fait de ces delx gens en somme,

5 Et comment ont fait retorner

Ung homme mort son esperit,

Ressusciter, aller, parler;

Ce cy oncques homme ne vit.

$$
\text { Malaquin. }
$$

Je m'y en vois tantost courir,

ro Je serai plus tost cy que la

Et ma boute[i]lle baisee sera

Avant que je voisse plus loing.

Pausa. Le messaige s'en va devers l'empereur

Corner. et luy dit

. . . . de vaillence

Nun beginnt die Uebereinstimmung beider Texte aufs neue.

Jubinal I, 76-77.

S. Pierre.

Je voy bien ce seroit trop fort;

Que de legier fust depecie

Que de legier fut despec(i)ee

9 Der Reim auf courir fehlt. 10. Ir. Vielleicht ist zu lesen: Et plus tost cy que la serai Et ma bouteille baiserai. 
Corde de trois cordons bastie: Je suis seul et vous estes trois.* Puis qu'il vous plaist donc je m'en vois.

Lors s'en voise et Jhesus ly veigne a l'encontre.

Pierres, bien soies tu venu!

S. Pierre a genoulz.

5 Sire Jhesus, et ou vas tu? Jhesus.

Pierres, Pierres, a Romme vois Pour mourir de rechief en crois.

Lors s'en retourne Jhesus sans plus dire.

\section{S. Pierre a genoulz.}

Je m'en revois; pardon, chier sire, J'apercois bien que voulez dire.

Lors s'en revoist a ses compaignons et die:

10 Chiers freres, quant je m'en aloie, Jhesucrist trouvai en ma voye

A qui demandé ou aloit;

Il me dit qu'a Romme venoit Pour estre encore en crois pendu.

15 A ces mos ay bien entendu Qu'il vouloit que je retournasse Et que ma vie en crois finasse; Sy ne l'osay oncques desdire.

Titus, Lucas, Clemens.

Sa volonte soit faicte, sire.
Corde de trois cordons cordee; Je suis seul et vous estes trois. Puiz qu'il vous plaist don je m'en vois.

Pausa. Saint Pierre s'en va et Dieu descent de paradis et luy dist quant il est a luy Deus :

Pierre, bien soies tu venu! Petrus.

$\mathrm{He}$, doulx Jhesus, et ou va[s] tu? 5 Deus.

Pierre, Pierre, a Romme vois

Pour morir de rechief en croix. Dieu s'en retorne en paradis et Saint Pierre s'en vient au millieu du champ et dit

Petrus:

Je m'en revois; pardon, chier sire, J'entent bien que vous voles dire.

Pausa. Saint Pierre torne aux apostres don il est parti et dit

\section{Petrus:}

Mes freres, quant je m'en aloie, Io Jhesucrist trouvé en ma voie, Luy demandé ou il aloit; Il me dist que a Romme venoit Pour estre encore(z) en croix pendu(s).

A cez moz j'ay bien entendu(s) I5 Qu'il vouloit que je retornasse Et que ma vie en croix finasse; Je ne l'ay volu escondire.

Clement, Titus et Lucas dient ensemble:

Sa voullente soit faicte, sire.

Hier hört die Uebereinstimmung beider Texte zunächst auf und tritt erst weiter unten noch einmal auf kurze Zeit ein. Im Anholter Bruchstück lautet die Fortsetzung folgendermafsen:

\section{Petrus.}

20 Clement, nostre chier filz en Dieu, Aies toujours devocion

* Titus, Lucas und Clemens; s. weiter oben. II trouvai hat die Hs., nicht trouvé, wie bei Jubinal steht

(Poewe 74).

20 Der Reim auf Dien fehlt. Vielleicht ist auf Grund der Stelle weiter oben (S. 83) zu lesen: Clement, nostre chier fils en Dieu, Qui tendrez apres moy mon lieu. 21 touriours. 
A Dieu qui souffrit passion Pour nous sauver et rachapter Hors de la pulentie d'enfer; Aies toujours en luy fiance

5 Et y mectes vostre esperance Et croies qu'il vous aidera.

Paulus.

Mes freres, qui Dieu aymera

Et de bon cuer le servira, Il le(s) mectra en paradis

Io En la gloire ou sont ces amis.

\section{Petrus.}

Mes chiers freres et mes amis, Temps est que nous nous departon De avec vous et nous en allon Paul et moy ung pou par la ville

15 Pour prechier la sainte evangile De Dieu comme il nous a commis; Ainxi le couvient, mes amis, Pour cunvertir et amender Le peuple et de erreur oster

20 Affin qu'il croie en nostre loy; Sy nous en allons sans deloy, $\mathrm{Ne}$ faisons plus cy demorance!

\section{Clement.}

Jhesucrist vous gart de grevance . . . . . . encombrement;

25 Jhesus, le roy omnipotent, Vous gart de mauvaiz encombrier!

\section{Lucas.}

A Dieu soies, pere treschi[e]r!

$\mathrm{Je}$ vous requier, peres, pour nous.

$$
\text { Petrus. }
$$

A Dieu soies, amys tresdoux!

$$
\text { Paulus. }
$$

30 Mes freres, a Dieu soies vous!

Pausa. Pierre et Paul s'en vont parmi le jeu et l'emperiere Noiron fait envoier querir ces tirans pour les prendre et dit a Agrippe le prevost

\section{Noiron:}

Sus, Agrippe, hativement!

Faites tost prendre ces pallars

Et les me livres a torment;

En mal feu d'enfer soit ilz ars.

3 s. Anmerk. + tourious. 13 l. D'avec. 19 s. Anmerk. 34 soit ilz ist vielleicht in soient zu ändern, doch ist soit eher als einsilbig gebrauchtes soient aufzufassen; vgl. nämlich 91,17 und 93,27 . 
Qui les laissera plus regnier Tout le peuple convertiront

Et nous mectront en grant dang[i]er

Et mon empire destruiront.

Paulinus senateur.

5 Tout le monde en leur loy mectront

Se ne les mectres a martire,

Et vostre empire destruiront.

Faictes les morir, treschier sire!

Domicien.

Se tost ne les faictes destruire;

Io Croies que vous desconfiront

. . . feront souffrir martire

Du peuple qu'il convertiront.

Noiron.

Par tous nos dielx ennuit morront.

Mais que les puicions atrapper,

16 Maint mal leur feray endurer

Ennuit et leur Dieu regnoier

Et cy mourront villainement.

Prevost, faictes tost vistement,

Envoies querir voz tirans

20 - De mal faire sont desirans -

Par le herault hastivement.

Agrippe le prevost.

Messagier, vien avant!

Tu es bien preux et saige;

Je te pri et commant

25 Que faces mon messaige.

Va moy tantost querir,

Je t'en pry, mes tirans,

De crestiens tenir

Ilz sont bien desirans.

30 Goulaffre et Marpault

Et Tornebroche ensemble,

Rifflart, le fort ribault,

Eulx quatre tous ensemble.

Bien seront martires

35 Chrestiens qui sans doubte

Nos dieux ont desprises

Et Mahomerie toute.

$\mathrm{Di}(\mathrm{s})$ leur(s) que point n'arestent

40 . . . . . . . . .

II Die ersten Buchstaben der Zeile sind nicht zu entziffern; es wird zu lesen sein: Et vos feront s. $m$. 13 nous dielx. 17 Der Reim auf regnoier fehlt. 36 Nous. 37 um eine Silbe zu lang; vielleicht ist Et zu uilgen. 
Et leur tormen...

[To]st et ynelle[ment].

Le messag[ier].

Sire, presentem[ent]

Je les . . .

5 Tost et hastive[ment]

$A$ vous je reven[drai].

Pausa. Le messagier $s$.

$$
\text { - - les tirans et } d
$$

Goulafre et Marp[ault],

Venes tost et gra[nt erre]

Si feres fort assau[t]

Io Et aux chrestiens gue[rre];

Avec vous Tornebroche

Que porte maint tor[ment],

Riflart, tous quatre en $t$ [roche]

Au prevost vistement

15 Apourtes voz . .

Et qu'il soient bien trai .

Pour partir le . .

A ces faulx crestien[s].

Goullafre.

$O$ nous les pourte. .

20 Nous sommes molt cru . .

Leurs testes coperon

Par dessoubs leurs.

Le messagier.

Ja nous ne leur faur. .

Certes faire de ma..

25 Mahon ont regnoi .

Marpault.

Mal leur est adjo.. .

Sy je les puis tenir

Je le[s] deshacherai

30 Et les feray mourir.

Tornebroche.

Se je les puiz tenir

Certes, nulluy n'en doubte,

Je les feray fonir

Et tous celx de leur roucte.

Rifflart.

35 A torment les mectray,

Nul de vous .... doubte,

Morir je les feray;

Je ne m'en faindray gouct[e]. kommt.

33 fonir wohl statt des gewöhnlichen fenir, da fouir kaum in Betracht 
Pausa. Les tirans s'en vont au ...

Corner. et le messagier les ma ...

et quant il luy sont...

Celuy qui trestout fait

Gart le prevost de Romme

Et honneur vous envoit,

C'est le grant dieu Mahomme.

5 A vous sommes venus,

Certes, sire prevost,

Nous sommes acourus,

C'est pour venir plus tost.

Agrippe le prevost.

Bien soies vous venus,

10 Vous quatre tous ensemble

Deulx garsons . . .

15 . . . . . en troche

. . . . . pourmenes

. . . . grant force

. . . . . . ement

20

. . . . . mes amis

$$
\text { vistement }
$$

annemis.

[G]oulaffre.

$$
\begin{aligned}
& \text {. . . . . . seront } \\
& \text {. . . . . . amere } \\
& \text {. . . . . coulx parront } \\
& 25 \text {. . . . . . et darriere } \\
& \text {. . . . tantost seront } \\
& \text {. . . . . [g]rant misere. } \\
& \text {. . . . efment mourront } \\
& \text { - . . mectrons en biere. } \\
& \text { [M]arpault. } \\
& 30 \text {. . . . leurs desrompre } \\
& \text {. . . . . . et darriere } \\
& \text { - . . . . lez mectre } \\
& \text {. . . . . a misere. }
\end{aligned}
$$

38 und 39 reimen nicht. Vielleicht ist $3^{8}$ zu lesen: qui m'avile (von aviler). 
[A]grippe le prevost.

. . . . toute la ville

. . . . . rien ne demeure

- . . que soit que ne meure

. . . es le soit foul ou nice

5 . . . ost je en feray justice.

[To]us les tirans dient ensemble:

. . . ne vous doubtes de rien;

[Par] [M]ahomet vous dictes bien.

. . [le]s tirans s'en vont parmi

. . le jeu ... saint Pierre commence a prechier. Petrus.

$[\mathrm{P}]$ etite et accipietis; pulsate et aperietur

[v]obis regnum celorum.

- peuple, entendes ma parolle,

[Ve]nes trestous a mon escolle,

ro . . vous feres grande sagesse;

- tendes ung pou sans paresse

- . parolles que vous veul dire

. . Jhesucrist nostre vray sire

- . sans peche le servira,

I5 [E]m paradis ou luy yra.

Helas, n'aies point de fiance

Es ydolles de grant villance!

[C]ar pour certain il ne voist goucte

- . uoist auxi null[u]i n'en doubte.

20 Riens ne puet ilz fere sans fa[i]lle;

Vous laisses le grain pour la paille,

Vous laisses de Dieu le service

Par vostre tresmauvais convice

. . . air a l'annemy

25 . . . . . ne demy.

Pausa. . . .

Noble empereur . .

Que t'avon amene;

Somes nous gens musarde,

Est ce bien ordonne?

30 Malsain feu d'enfer m'arde

Sy ne me suis penes;

Ilz font l'arrieregarde

Comme preux et cenes.

Marpault.

Trouve l'avon preschant

35 La enmy une rue;

181. Car pour certain ne voient goucte, aber in der folgenden Zeile läfst sich schwer eine befriedigende Lesart herstellen. 201 . Riens ne puent fere sans faille. 26 das fehlende Wort wird esgarde oder regarde sein. $30 \mathrm{Mal}$ sin. 
Il(z) l'aloit empeschant,

La loy sans retenue;

La hault em plain merche

Fesoit leur preschement,

5 . . . soit de peche

Et mis a mort vrai(e)ment.

Tornebroche.

C'est ung larron pruve

Et si est crestien;

Prechant l'avons trouve

Io De dieu nazarien.

Ilz ont tresmal ouvre,

Donc batus seront bien;

En moy ilz ont trouve

Plus aspre que chien.

Noiron.

15 Bien soies arives;

Vous estes mes amis,

Sy me soist aprouches

Ces felons annemis.

Je veul d'eulx enquerir

20 La cause et la raison

Pour quoy firent mou[rir]

Ainsi maistre Symon.

Pierre, dy moy pour $q$. .

La maniere et coment

25 Tu fis mourir Symon

Ainssi villainement.

Petrus.

Noiron, je te diré,

Tout son gouvernement

En enfer est tiré

30 A paine et a torment;

Car dieulx il se disoit,

Donc estoit menterie;

Es cielx voller voulloit

Par son enchanteric.

35 Les diables l'ont deceu

Qu'il a toujours servis,

Tant ont fait que l'ont eu

A faire a leur devis.

Tous celx qui serviront

40 Jhesu devotement

Et du cuer l'ameront

Sy aront sauvement;

I la loit. 14 zu kurz; vielleicht Plus aspre que nul chien. 17 ist nur verständlich, wenn soist als Entstellung von einsilbig gebrauchtem soient angenommen werden darf. 36 Que la touriours. 
Em paradis iront

Sans paine et sans torment.

Se aultrement ilz le fo[nt]

Yront a dampnem[ent].

Noiron.

5 Blafemes tu en cest ...

Nos dieux Pierre apo...

Qui fortrois celx...

Quant nostre amy mai ...

As fait mourir . . .

10 Et qui nous fais ...

Die nun folgenden Verse entsprechen wieder einer Stelle bei Jubinal. In der Scene, die der letztern dort vorhergeht, tritt gleichfalls wie im Anholter Bruchstück Agrippa mit seinen Henkersknechten auf, die jedoch, einen (Riflart) ausgenommen, andre Namen führen. Sie treiben mit den beiden Aposteln Petrus und Paulus ihr frevles Spiel und vergreifen sich sogar thätlich an ihnen. Zuletzt werden die Apostel auch dort zu Nero geführt; mit den letzten Worten von dessen Anrede an Petrus beginnt die Uebereinstimmung beider Texte.

$$
\begin{gathered}
\text { Jubinal I, 80-8I. } \\
\text { Neron. }
\end{gathered}
$$

Anholter Bruchstück.

Tu fais merveilles, tu fais rages, ... que .. le sache

Tu es(t) tout plain de malefice(s), . soit lies en celle estache

Sy fault faire de toy justice; . es de mort sont vraiement

Raison, les drois, les loys le veulent. . . son les loiz les droiz le veullent.

$$
\text { S. Pierre. }
$$

Petrus.

15 Raison ne drois ne loys ne veulent . son ne droit ne loiz ne...

Que ceulz qui tenir verite seulent. Ix qui tiennent veritez

- puiz l'eure que furent nez

En cuer, en bouche, en meurs, en vie, . . ont tous les jours de leur vie

Aient ne mal ne vilenie; $\quad \ldots$ ne mal ne villainie;

20 Mais ceulz qui aiment faussete . 2 celx qui aiment faulcete

Doivent avoir meschancete, . . aient avoir mechanssete

Comme Symon vostre enchanteur, .. Symon vostre enchanteur

Faulz, renoie et fol vanteur, _. regnoie et faulx venteur

Qui Dieu tout puissant se fesoit .. eu tout puissant ce tenoit

25 Qui es cieulx voloit, ce disoit; . . s celx voller ce disoit

Mais non fesoit, pour voir estoient . . non fasoit pour voir s'estoient

Dyables d'enfer qui le portoient, . . les d'enfer qui l'emportoient

3 l. S'aultrement. 6 Nous. 7 fortrois, deutlich, wohl Fehler für fortrais von fortraire.

12 Hs. est (Poewe 74). 16 zu lang; vielleicht ist verte $\mathrm{zu}$ lesen; $\mathrm{vgl}$. 18 tout les jours. weiter oben S. 79.24 puissans. 
Qui malgre eulz cheoir le lesserent . ulgre eulx choir le laisserent

Tout vif et les os ly froisserent, . . vif et les oulz luy froicerent

Quant il pleust a Dieu qui tout puet, . . il(z) pleust a Dieu qui tout peult

Qui tout gouverne, qui tout muet, . . gouverne et tout meult

5 Qui n'eut oncques commencement .. ques n'out commencement

$\mathrm{Ne}$ ja n'ara definement.

.. n'aura definement

.. voir que en temps et en lieu

. grant doulceur le vray Dieu

Par sa grant doulceur le vray Dieu

... sauver il devint homme

Pour sauver home devint homme,

1o Qui s'estoit forfait par la pomme,

. . forfait pour la pomme

Et en la crois laide et honteuse . . oix laide et honteuse

Souffrit mort dure et engoisseuse, . . mort tresengoiceuse

Toutevois bonne et profitable. .. es sy fut prufitable.

\section{Neron.}

$[\mathrm{N}]$ oiron.

Tes toy, vilain, ce n'est que fable. ...... n'est que fable

I5 Et toy, Pol, que vas tu lisant?

S. Pol.

Sire, je vois tout ce disant.

Die Fortsetzung des Bruchstücks bis zum Schlusse ist, was den Wortlaut anlangt, von dem Texte bei Jubinal völlig abweichend. Inhaltlich herrscht insofern Uebereinstimmung, als auch dort das Gespräch zwischen den Aposteln einerseits und Nero bzw. Agrippa und deren Henkersknechten andrerseits noch eine Weile andauert. Es folgt dann dort der Märtyrertod der Apostel, der Selbstmord Neros und zum Schlusse die Wahl des h. Clemens zum Papste.

$$
\begin{aligned}
& \text { - . . - decevable } \\
& \text { - . e vois desprisent } \\
& \text { - . . suiz empereur } \\
& 20 \text {. . . noble du monde } \\
& \text { - . . vas a doleur } \\
& \text { - [en]chanteur de monde } \\
& \text {. . . . hastivement } \\
& 25 \text { - . - . - ost prestement } \\
& \text {. . . . je desire. } \\
& \text { [A]grippe. } \\
& \text { - soist mis en celle estasche } \\
& \text { - } 1 \text { sont en voz mains coulles } \\
& \text { - . et cuir leur arache } \\
& 30 \text {. . battus et triboulles } \\
& \text { - . qu'il aist mainte tache }
\end{aligned}
$$

I cheoir einsilbig; vgl. weiter oben S. 8I. 10 s'estoit] jestoit Hs. Der ganze Vers fehlt in dem Texte von Jubinal. Vgl. Poewe 74 .

22 Statt de monde ist vielleicht immonde zu lesen. 27 scheint in soist einsilbig gebrauchtes soient zu stecken; ebenso aist $31=$ aient.
10 pour wohl verderbt aus par, wie die Hs. der Bibl. Sainte-Geneviève hat. . 
- . . ges de fer foulles

- . [m]out bien veul qu'on le sa(i)che

. . dieulx ait ainxi voulles.

- . tirans les despoullent et les

- . en l'estasche et Goullafre dit

. . . . renderé

5 . . . cele chiffle

. . . . . teré

. . . mainte bifle

. . . . acheré

- . . e je sifle

Io . . . . eray

. . . . . tise

. . . . cest euf

. . . . . . se

$\mathrm{Je} \cdot \cdot \cdot \cdot \cdot \cdot \cdot$

Petrus.

15 Prevost, tu dis grant menterie;

Je ne suis mie enchanteur.

Agrippe le prevost.

Garson, m'appele[s] tu manteur

En despit de trestous nos dieulx

De Cahu et de ces autielx

20 Et de toute lcur quirielle?

Je vous dorroy ceste merelle

Pour avoir vers moy tel langage.

Tirans, ce fellon, plain de rage,

Me soit deslie tout en l'eure

$25 \mathrm{Si}$ soit mene sans nul demeur[e]

En ma chaltre la plus obscure,

Menes le moy par grant ledure!

Dictes au chartrier que luy mande

Qu'il n'ait ne vin ne viande

30 Nullement ne point de lumiere

Ne nulle rien qui luy affiere;

Alles et faictes vistement.

Pausa. Les tirans le deslie[n]t et dit

Goullafre:

Or avant, villain, maintenant,

Estroitement serez tenu;

35 L'en vous fera si grant esbru

Que le deable vous aidera.

Marpault.

Par Mahon, l'en vous hous[s]era.

Or tenes! mectes ceste bride!

1. Que il.

18 nous dieulx. 27 Für le scheint l'y zu lesen zu sein. 29 zu kurz 
Tornebroche.

Faulx chrestien, sus! ride, ride!

Tu mor... il n'y a. . .

Ce cy auras pour te froter.

Rifflart.

5 Des miches de son abaie

- . sus luy dieu le main. .

$\mathbf{M}[\mathrm{a}]$ hon luy doint fievre quartai[ne]!

Pausa.

Goullafre.

Chartrier, vez cy que vous amai[ne]!

Mahon le met[e] en mal[e] estraine;

Io Noiron, emperiere de Rome,

Si le hait plus que nul aultre homme.

Mes le moy dedens loger la

Et garde qu'il ait fain aux de[ns].

Marpault.

Chartrier, gardes bien . . .

15 Serre luy desoubz la grant tra..

D'un fer pesant que ne s'en fu[ie],

Car il joue d'enchanterie.

Tornebroche.

N'a il pas fait ressuciter

Ung home mort pour baptiser

20 Tout le peuple de la cite?

\section{Rifflart.}

ll(z) est vray, par ma verite,

Il [l']a fait par enchantement.

Le chartrier.

Maistre Pierre, passes avant!

Vous seres loge pour sepmaine;

25 Jupin vous doint fievre carta[ine].

Quant aves conte, dit Noiron,

Et fait mourir maistre Simon,

Vous ne feistes onc tel foulie.

Or ne vous en soucies. .

30 Je le gouverneray tresbien.

Goullafre.

De luy . . . . . .

Tornebroche.

[Je] pry Mahon que le feu l'arde

- . est enchanteur repruve.

8 Chartries. I I l. Sil $(=\mathrm{Cil})$ le. 12 dedens ist durchgestrichen, scheint aber am Schlusse eingesetzt werden zu müssen. 13 qui lait. 16 qui statt que. $26-27$ scheinen nicht völlig richtig zu sein. 28 ong. $29 \mathrm{Um}$ den Vers herzustellen könnte Ore gelesen und hinter soucies das Wort mie eingesetzt werden. Aber auch dann würde noch das Reimwort zu tresbien fehlen, $\mathrm{da}$ das Reimwort zu l'arde (V. 32) doch wohl garde ist. 
Rifflart.

- dit vray, par ma loiaulte,

- est tout plain d'enchanterie.

Le chartrier.

[Je] luy metray si dure vie

[Qu']il ne aura garde d'enchanter.

Les tirans s'en retournent

au prevost et dient tous ensemble:

5 . - chartremez si dit vraiement

- grief mourra vilainnement.

Agrippe le prevost.

C'est bien dit, par les dieulx du temple

- autre y prandront exemple.

$[\mathrm{Pa}]$ ulus, enten ung poy a moy!

[Veu]lx tu toujours estre en esmoy

- . ter ta belle jeunesse

- . es et de belle prouesse,

. . . ta belle juvence

- . dy en ceste presence

15. de rien tu t'aviseras

- Mahomet aymeras

- j'ay trop grant pitie de toy

- . avant et cy me croy

- sacriffier noz dielx

20 . . . la terre et les cielx

- . te feray grant honneur

. . . estray .. l'empereur

- . richesses te dorroy

25 . . coronne de fin or

- abandonne mon tresor

. . honnorablement.

Paulus.

- me conseilles mallement

. . celuy qui fit cest jour

30 . . tresmauvaiz conseilleur

- . ton dieu Mahomet ne vault

- aultres ains dire fault

- . diables que vous croies

- vous ont ainxi enchantes.

35 . . sont faulx qui les sacrifient

- qui envers eulx se humilient

4 1. n'aura. Das Reimwort zu enchanter fehlt. In der nun folgenden Bühnenanweisung hat die Hs. sans für s'en. 5-6 vielleicht Enchartrene, si di vraiment, Grief mourra et vilainnement. 10 touriours. 30 ist vielleicht conseillour einzusetzen. $\quad 31-34$ sind vielleicht folgendermafsen berzustellen: Riens ton dieu Mahomet ne vault Ne les aultres, ains dire fault Ce sont diables que vous croies Qui vous ont ainxi enchantes. 


$$
\begin{aligned}
& \text {. . me gart que point je n'y croye } \\
& \text {. . tel mal pruvez je soie } \\
& \text {. dieu Appolin rien ne vault } \\
& \text {. Sogolin au besoint fault } \\
& \text { 5 . ont ne force ne povoir. } \\
& \text {. Agrippe le prevost. } \\
& \text { [Paul]us, tu ne dis mie voir } \\
& \text {. . llin si peult trestout faire } \\
& \text { Sogolin . . tout desfaire } \\
& \text {. . . tost de toy aviser } \\
& \text { 10. se aourer les vourres } \\
& \text {. . . [o]bair leur vouldras } \\
& \text {. . . maint mal souffriras } \\
& \text {. . . . fais par verite } \\
& \text {. . . . mourras a vilte } \\
& \text { 15. . ton Jhesus desdire } \\
& \text {. . . . hon nostre sire } \\
& \text {. . . . feras que saige. }
\end{aligned}
$$

10 wird vourras statt vourres zu lesen, ferner aber aourer ans Ende der Zeile zu setzen sein.

\section{Anmerkungen.}

S. $77^{\text {a }}$ V. 1. nient ist bier und S. $7^{\text {a }}$ V. 8 einsilbig gebraucht, was auch für andre Stellen des Textes von Jubinal angenommen werden darf; vgl. Nient meins je vueil que ce faciez I, 40; Nient plus que feroit une truye III; Nient mains vous faites tout possible 237; C'est nient, je ne puis prendre somme 250; Nient mains il veult qu'entierement 296; ferner 198, wo nient im Reime mit vient steht. An mehreren Stellen hat der Text néant oder néent: Cé doncques qu'il a de néant fait I, 45; Qui de néent le monde crea 62; Qui de néant crea tout et fist 111 ; Néant mains, bele suer, regardez 299; allein sämtliche Verse sind um eine Silbe zu lang und vermutlich durch Einsetzung der einsilbigen Form $z u$ verbessern.

S. 78 a V. 4. sa bezieht sich auf l'anemis.

Ebd. V. 9. Littré, Dict. 3, 24 weist darauf hin, dafs früher neben immuable auch immutable gesagt wurde, und belegt dieses durch das Beispiel, das Jubinal I, 194 steht.

Ebd. V. 22 scheint so belassen und paisant als zweisilbig genommen werden zu müssen wie auch in dem Verse bei Jubinal I, 123 Or suz, liez moy ces paisans! Diese zweisilbige Geltung des Wortes kommt nach Littre, Dict. 3, 1022 noch im 17. Jabrh. bei guten Schriftstellern vor.

Edb. V. 35. mais, Femin. maise "schlecht". Viele Belege schon bei Foerster, Venus la deesse zu $179^{2}$.

S. $78 \mathrm{~b}$ V. 22. pencez für pensez. c für $s$ (ss) begegnet häufig im Anholter Bruchstück: $c e=$ se $79,12,20 ; 80,11 ; 92,24 ;$ pugnice 80,16 ; ces S. 86 Bühnenanweisung; puicions 87,15 ; cenes 90, 33; faulcete 92, 20; froicerent

Zeitschr. f. rom. Phil. XXVI. 
93, 2; tresengoiceuse ebd. 12 . Umgekehrt $s$ (ss) für $c$ in se $=c e 80,28$; dessoit ebd. 24; si 82, 26; meschanssete 92, 21 .

S. $78 \mathrm{~b}$ V. 33. envellimeur = envenimeur "Vergifter".

$\mathrm{S} .79^{\mathrm{b}}$ in der Bühnenanweisung sind die Worte grisogoulos guligoleste wohl eine unverständliche Zauberformel.

S. 79a V. I3. sanglant „schmählich“. Das Wort ist in dieser Bedeutung sehr beliebt bei Arnoul Greban, Mystère de la Passion; s. das Glossar.

Ebd. V. 15. contrarguer „Gegengründe vorbringen, widerlegen, widerstreben" wird von Godefroy nur durch eine einzige Stelle belegt (aus der Advocacie Nostre Dame, Hs. in Evreux), findet sich jedoch bei Jubinal auch I, 248 sowie im Lothringischen Psalter. An der letztern Stelle heifst es Psalm 37, 13-14 Et je comme sours ne les voloie escouteir ne oyr et comme muelz n'ovroie point ma bouche; Et suis fais comme home que riens n'oyoit et qui en sa bouche n'ai perolle pour lui revengieir ne contre-argueir. Die entsprechende Stelle lautet in der Vulgata: Ego autem tamquam surdus non audiebam, et sicut mutus non aperiens os suum. Et factus sum sicut homo non audiens et non habens in ore suo redargutiones.

S. $80 \mathrm{~b}$ V. 25 wird statt surtrait zu lesen sein sotrait oder soutrait, wie $A$ hat; denn surtraire heifst "verführen".

Ebd. V.26. vice begegnet auch sonst gelegentlich als Femininum; so an einer bei Littré, Dict. 4, 2476 angeführten Stelle.

S. $8 \mathrm{I}$ a V. 3. Statt coyssy ist vielleicht croyssy ,zerbrochen, zermalmt“ zu lesen, wenn nicht eine Infinitivform coissir neben der üblichen coissier (neufr. cosser) "stofsen" angenommen werden darf.

Ebd. V. 5 courir und V. 8 baler, nicht venir und voller in B wird die richtige Lesart sein, vielleicht auch schon deshalb weil der Verfasser des Stückes den leoninischen Reim liebt. S. die zahlreichen Beispiele bei Poewe 36 .

Ebd. V. 14. Häufiges altfr. Sprichwort; vgl. Tobler, Proverbe au vilain zu 21, 7, Berger, Canchons und Partures des A. de le Hale I, 43.

Ebd. V.19. vendus congruiert im Geschlechte nicht mit den vorhergehenden Substantiven. Ein solcher Fall begegnet auch sonst im Altfr. nicht ganz selten, wie Tobler zeigt, Vermischte Beiträge I, 193.

Ebd. in der Bühnenanweisung bezeichnet champ die eigentliche Scene der Bühne; vgl. Petit de Julleville, Les Mystères I, 388, 398 und II, 154. Ebenso bei Jubinal I, 92, 94, 95 ; II, 84, 93 u. s. w.

S. 82 V. 24. gouverner hier und S. 95 V. 30 "Sorge tragen, besorgen".

S. $82^{\mathrm{b}}$ V. $3 \mathrm{I}$ hat die Hs. deutlich eau; die einsilbige Form kann aber für die Zeit kaum angenommen werden.

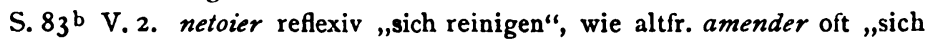
bessern" heifst. ? Schwerlich. Die Stelle wird verderbt sein und $\mathbf{A}$ das Richtige bieten.

Ebd. V. 24 ist die Lesart gleichfalls kaum die ursprüngliche, eher die des nebenstehenden Textes, während im folgenden Verse inpaciens vor imparfais den Vorzug verdient.

Ebd. V. 26. faire essamplaire kommt auch im Testament von Jean de Meun vor (Godefroy).

S. 84 V. 4 . delx d. h. deus (duos); vgl. oulz $=$ os S. $93^{\text {b V. }}$. Solche 
Schreibungen besprechen Foerster, Venus la deesse zu $85^{\mathrm{b}}$ und Suchier, Aucassin und Nicolete' zu 2, 39.

S. 85. Wegen der hier berichteten Legende von der Begegnung des Apostels Petrus mit Christus vgl. die Acta Sanctorum mensis julii I, 304 (De Sanctis Processo et Martiniano): Beatissimo autem Petro Apostolo, cujus pedem attriverant compedes ferrei, cecidit fasciola apud sepem in via nova: cumque venisset ad portam Appiam, vidit Dominum fesum Christum, eumque agnoscens, dixit illi: Domine, quo vadis? Et Dominus: Romam, inquit, redeo, ut itcrum crucifigar: tu autem Romam revertere, rediitque Petrus Romam mane, et milites tenuerunt eum.

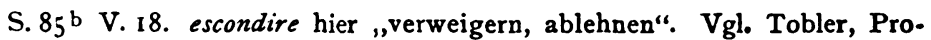
verbe au vilain $\mathrm{zu} 95, \mathrm{I}$.

S. 86 V. 3. Der um eine Silbe zu lange Vers ist vielleicht dadurch zu bessern, dafs für pulentie das von Godefroy zweimal belegte pulenteur eingesetzt wird.

Ebd. V. 14 hat die Hs. deutlich Paul, nicht Pol, wie sonst die richtige altfr. Form heifst, cbenso auf der nämlichen Seite in der Bühnenanweisung.

Ebd. V. 15. evangile, meistens Masculinum, aber auch Jubinal I, 29 sowie bei A. Greban, Mystère de la P. V. 16007 und 32618 desgl. Ztschr. $\mathrm{XXV}, 106$ als Femininum gebraucht, findet sich als solches noch im 17. Jahrh.; s. Littré, Dict. 2, 1543 und D. et H., Le seizième siècle en France I, 248.

Ebd. V. 19 könnte gelesen werden Le peuple et si d'erreur oster, aber eher mufs es vielleicht heifsen Le peuple et de s'erreur oster. Der altfr. Gebrauch den Vokal von ma, ta, sa vor vokalischem Anlaut zu elidieren begegnet auch weiter oben S. 83 V. 7 in s'ame und findet sich noch an einer Reihe andrer Stellen bei Jubinal, wie Poewe S. 25 zeigt, ${ }^{1}$ ja häufiger als der neufr. Gebrauch.

S. 86 und 90 in der Bühnenanweisung ist unter jeu die Bühne zu verstehen; vgl. die die Aufführung der grofsen Passion von Jean Michel betreffende Stelle bei Petit de Julleville, Les Mysteres II, 50: Pour mieulx commancer et avoir sillence, si l'on voit qu'il soit expedient, sera dicte une messe ou (au) jeu, sur ung autel honnestement droissé.

S. 87 V. 37. Mahomerie bedeutet hier nicht wie gewöhnlich eine Moschee, sondern den Muhamedanismus. So auch an mehreren Stellen bei Godefroy.

S. 88 V. 13. troche "Bündel, Vereinigung“ (en trocke „vereint, zusammen"; s. Godefroy), das S. 89 V. 15 deutlich von der Hs. geboten wird, scheint auch hier am besten in den Reim und in den Zusammenhang zu passen.

S. 89 V. 4. Mahomme, hier durch den Reim gesichert, wohl entstanden durch Einfluss von homme (hominem).

Ebd. V. 15 und 17. troche: force wird $z u$ belassen sein. Aehnliche ungenaue Reime gestattet sich häufig besonders Benoit de Sainte-More; s. die Schrift von Settegast über diesen Dichter S. 10; vgl. ferner viaige: charge Ztschr. XIX, 210, malade: guarde Marie de France, Lais S. 130.

$1 \mathrm{Zu}$ den dort angeführten Beispielen gesellt sich m'office I, II und 60, an beiden Stellen als Femininum gebraucht wie bei A. Greban, Mystère de la Passion V. 23151 De 'office qu'il t'a commise. Als Femininum findet es sich noch im 16. Jahrh.; s. Darmesteter et Hatzfeld a. a. O. I, 249. 
S. 90. Die bekannte Stelle aus der Bergpredigt (Matth. 7, 7), auf die hier Bezug genommen wird, lautet in der Vulgata: Petite, et dabitur vobis; quaerite et invenietis; pulsate et aperietur vobis.

Ebd. V. I5. ou d. h. o (od) "mit".

Ebd. V. 23. convice „Beschimpfung“; s. Godefroy.

Ebd. V. 30 vgl. Ebeling, Auberee zu V. 518.

S. 91 V. 4. leur ist Dativ.

Ebd. preschement „Predigt“; vgl. Jubinal I, 249, A. Greban, Mystère de la Passion 19585, Godefroy.

S. 93a V. 10. Qui s'estoit forfait par la pomme ,der sich durch den Apfel (durch den Bils des Apfels) vergangen hatte".

Ebd. V. 13. Toutevois merkwürdige Form des Uebergangs vom altfr. toutes voies, toutevoies zum neufr. toutefois.

S. 94 V. 5. chif(f)le „Pfeife, Auspfeifen, Verspottung“ besteht im Altfr. neben chufle, wie im Provenzal. chifla neben chufla; vgl. Diez, E. W. (ciufolo). Beide Formen belegt Godefroy.

Ebd. V. 7. bife, bifle „Ohrfeige“, das dieser nicht bietet, wird aus dem üblichen bufe durch Einflufs von altfr. bife, auch bifle (Name eines Kleiderstoffes) entstanden sein, wie umgekehrt neben diesem letztern auch bufe vorkommt. Die Einschiebung des $l$ wie in estofle: (Christofle) Jubinal I, 186.

Ebd. V. 20. quirielle „Litanei“" heifst hier wohl „Gefolge, Anhang".

Ebd. V. 2r. merelle hier „Faustschlag“; vgl. Jubinal I, 121 : Tien, apostat, ceste merele!, A. Greban, Mystère de la Passion 20964-5: Or nous prophetise, Thesus, Qui t'a baille ceste merelle.

Ebd. V. 25 ist demeure als Masculinum gebraucht; doch scheint die Stelle nicht ganz unverdächtig.

Ebd. V. 26. chaltre = chartre; Dissimilation wie in prov. albre (arbor), polpra (purpura).

Ebd. V. 35. Die Bedeutung von esbru, das deutlich von der Hs. geboten wird, ist mir zweifelhaft, wenn es nicht etwa für esbrui steht und zu esbruire, esbruir „Geräusch machen" (s. Godefroy) gehört. $u$ für ui kommt zuweilen im Reime vor: us (ostium) : plus Bartsch, Chrest. ${ }^{5}$ 144, 34, Vie de Saint-Gilles V. 105, : sus Marie de France, Lais S. 125.

Ebd. V. 37 hat die Hs. housera, allein das Richtige wird houssera sein von housser, Ableitung von houx „Stechpalme“. Das Verbum heilst neufr. zwar nur noch „abstäuben", altfr. aber auch „mit Ruten peitschen“; s. die Stellen bei Godefroy und Littré, Dict. 2, 2057.

S. 95 V. 1 . ride wohl Imperativ von rider „reiten“ in der Bedeutung eines Zurufs: vorwärts!

Ebd. V.9. Mahon le mete en male estraine „M. bringe ihn in eine schlimme Bescherung hinein, beschere ihm etwas Schlimmes!“

S. 97 V. 4 und 8. Sogolin ist deutlich in der Hs. zu lesen; um welchen Namen es sich jedoch handelt, bleibt zu untersuchen.

Hugo Andresen. 